\title{
Stability of symmetric systems under hyperbolic perturbations
}

(Dedicated to Professor Rentaro Agemi on his sixtieth birthday)

\section{Tatsuo Nishitani}

(Received April 30, 1996)

\begin{abstract}
Let $L(x)$ be the symbol of a $m \times m$ symmetric first order hyperbolic system with real constant coefficients. The range of $L(x)$ is a subspace, containing a positive definite $L(\theta)$, in the linear space of dimension $d(m)=m(m+1) / 2$ of all $m \times m$ real symmetric matrices. We study a hyperbolic perturbation $\tilde{L}(x)=L(x)+R(x)$ of $L(x)$, that is $R(x)$ is $O\left(|x|^{2}\right)(x \rightarrow 0)$ which is real analytic and all eigenvalues $\lambda$ of $\tilde{L}(x+\lambda \theta)$ are real near the origin. We prove that if the dimension of the range of $L(x)$ is greater than $d(m)-m+2$, then generically, every such hyperbolic perturbation is trivial, namely there are real analytic $A(x), B(x)$ near the origin with $A(0) B(0)=I$ such that $A(x) \tilde{L}(x) B(x)$ becomes symmetric. When $m=3$, the same conclusion holds if the range is greater than 3 .
\end{abstract}

Key words: hyperbolic perturbation, symmetric system, non-degenerate.

\section{Introduction}

Let

$$
\mathcal{L}(x)=\sum_{j=1}^{n} A_{j} x_{j}, \quad x=\left(x_{1}, \ldots, x_{n}\right),
$$

where $A_{j}$ are real symmetric $m \times m$ matrices which are linearly independent. Since we are interested in hyperbolic systems we assume that $\mathcal{L}(\Theta)$ is positive definite with some $\Theta \in \mathbf{R}^{n}$. We may suppose that $\mathcal{L}(\Theta)=I$ considering $\mathcal{L}(\Theta)^{-1 / 2} \mathcal{L}(x) \mathcal{L}(\Theta)^{-1 / 2}$. The range $\mathcal{L}=\left\{\mathcal{L}(x) \mid x \in \mathbf{R}^{n}\right\}$ of $\mathcal{L}(x)$ is a linear subspace in $M^{s}(m, \mathbf{R})$, the space of all real symmetric $m \times m$ matrices. Note that the range contains the identity $I$ and of $n$ dimensional because $A_{j}$ are linearly independent.

We study the symbol $\mathcal{P}(x)$ of a hyperbolic system which is close to $\mathcal{L}(x)$ near $x=0$;

$$
\mathcal{P}(x)=\mathcal{L}(x)+R(x)
$$


where $R(x)=O\left(|x|^{2}\right)$ as $x \rightarrow 0$ which is real analytic near the origin and all eigenvalues $\lambda$ of $\mathcal{P}(x+\lambda \Theta)$ are real near $x=0$.

By Theorem 4.2 in [9], every hyperbolic perturbation is trivial if the dimension of the range $\mathcal{L}$ is maximal, that is $n=m(m+1) / 2=d(m)$ in the sense that there are real analytic $A(x), B(x)$ defined near the origin with $A(0) B(0)=I$ such that $A(x) \mathcal{P}(x) B(x)$ becomes symmetric. Our aim in this note is to study symmetric systems $\mathcal{L}(x)$ whose range have dimension less than $d(m)$.

Theorem 1.1 Assume $d(m)-m+3 \leq n \leq d(m)$. Then in the $(d(m)-$ $n)(n-1)$ dimensional Grassmannian of $n$ dimensional subspaces of $M^{s}(m, \mathbf{R})$ containing the identity, the subset for which hyperbolic perturbations are trivial is an open and dense subset.

Here we have identified a symmetric matrix $\mathcal{L}(x)$ with its range $\mathcal{L}$ because the assertion is independent of linear changes of coordinates $x$.

In Section 2, reexamining the proof and the hypotheses of the above mentioned result in [9] we show that: Let us denote by $S_{\mathcal{L}}(x)$ the linear map sending a $H \in M^{s}(m, \mathbf{R})$ with zero diagonal elements to an anti-symmetric $[\mathcal{L}(x), H]$. Let

$$
\operatorname{det} S_{\mathcal{L}}(x)=\prod_{j=1}^{s} g_{j}(x)^{r_{j}}
$$

be the irreducible factorization of $\operatorname{det} S_{\mathcal{L}}(x)$ in $\mathbf{R}[x]$. Then assuming that

$$
\left\{x \mid g_{j}(x)=0\right\}, 1 \leq j \leq s, \text { contains a regular point }
$$

and that every characteristic of order less than $m$ of $\mathcal{L}(x)$ is non-degenerate (see Definition 2.1) we can conclude that all hyperbolic perturbations are trivial (Theorem 2.1).

To check these two conditions, in Section 3, we study characteristics of $\mathcal{L}(x)$ and we prove that, in the Grassmannian of $n$ dimensional subspaces of $M^{s}(m, \mathbf{R})$ containing the identity, the subset for which every characteristic of order less than $m$ is non-degenerate is an open and dense subset (Proposition 3.3).

In Section 4, in this Grassmannian of $n$ dimensional subspaces, we show that the set for which the condition (1.2) is fulfilled is an open and dense subset if $n \geq d(m)-m+3$ (Proposition 4.1).

The last restriction on $n$ comes from purely technical reasons in proving 
Proposition 4.1 and it is plausible that it could be weakened. Indeed, if $m=3$, Theorem 1.1 holds for $n \geq 4$ :

Theorem 1.2 Assume that $m=3$ and $4 \leq n \leq 6=d(3)$. Then in the $(6-n)(n-1)$-dimensional Grassmannian of $n$ dimensional subspaces of $M^{s}(3, \mathbf{R})$ containing the identity, the subset for which hyperbolic perturbations are trivial is an open and dense subset.

The proof will be given in Section 5 . We can find detailed studies on the structure of 6-dimensional Grassmannian of 4-dimensional subspaces of $M^{s}(3, \mathbf{R})$ containing the identity in Theorems 3.5 and 3.6 in [4].

\section{Non-degenerate characteristics}

We first make precise the notion of non-degenerate characteristics of order greater than two (see [8], [9]). Let $\mathcal{P}(x)$ be a real analytic function with values in $M(m, \mathbf{R})$, the set of all real matrices of order $m$, defined near the origin of $\mathbf{R}^{n}$ with coordinates $x=\left(x_{1}, \ldots, x_{n}\right)$. Let $x=\bar{x}$ be a characteristic of $\mathcal{P}(x)$, that is $\bar{x}$ is a zero of $\operatorname{det} \mathcal{P}(x)$. Assume that

$$
\operatorname{Ker} \mathcal{P}(\bar{x}) \cap \operatorname{Im} \mathcal{P}(\bar{x})=\{0\} .
$$

In this case we can define the localization $\mathcal{P}_{\bar{x}}(x)$ of $\mathcal{P}(x)$ at $\bar{x}$ as follows (see Definition 3.1 in [8], see also [10], [1]). The assumption (2.1) identifies $\operatorname{Coker} \mathcal{P}(\bar{x})$ and $\operatorname{Ker} \mathcal{P}(\bar{x})$. Since $d \mathcal{P}(x)$, the differential of $\mathcal{P}$ at $\bar{x}$, is a well defined map going from $\operatorname{Ker} \mathcal{P}(\bar{x})$ to Coker $\mathcal{P}(\bar{x})$ then the map followed by the canonical map to Coker $\mathcal{P}(\bar{x})$ is identified with a map $\operatorname{Ker} \mathcal{P}(\bar{x}) \rightarrow$ $\operatorname{Ker} \mathcal{P}(\bar{x})$, which is the localization $\mathcal{P}_{\bar{x}}(x)$. For later references we give a representation of $\mathcal{P}_{\bar{x}}(x)$ in local coordinates. Set $s=\operatorname{dimKer} \mathcal{P}(\bar{x})$. Let $\left\{v_{1}, \ldots, v_{s}\right\}$ be a basis for $\operatorname{Ker} \mathcal{P}(\bar{x})$ and let $\left\{\phi_{1}, \ldots, \phi_{s}\right\}, \phi_{i} \in\left(\mathbf{C}^{m}\right)^{*}$ be linearly independent and vanish on $\operatorname{Im} \mathcal{P}(\bar{x})$ such that $\left(\left\langle\phi_{i}, v_{j}\right\rangle\right)=I_{s}$. Then $\mathcal{P}_{\bar{x}}(x)$ is given by

$$
\left(\left\langle\phi_{i}, \mathcal{P}(\bar{x}+\mu x) v_{j}\right\rangle\right)=\mu\left(\mathcal{P}_{\bar{x}}(x)+O(\mu)\right)
$$

as $\mu \rightarrow 0$.

Definition 2.1 Let $x=\bar{x}$ be a characteristic of $\mathcal{P}(x)$. We say that $\bar{x}$ is non degenerate if the following conditions are verified;

(1) $\operatorname{Ker} \mathcal{P}(\bar{x}) \cap \operatorname{Im} \mathcal{P}(\bar{x})=\{0\}$

(2) $\operatorname{dim}\left\{\mathcal{P}_{\bar{x}}(x) \mid x \in \mathbf{R}^{n}\right\}=s(s+1) / 2$ with $s=\operatorname{dimKer} \mathcal{P}(\bar{x})$, 
(3) $\mathcal{P}_{\bar{x}}(x)$ is diagonalizable for every $x$.

We call $s$ the order of the characteristic $\bar{x}$.

We return to $\mathcal{L}(x)$ mentioned in Introduction. By a linear change of coordinates $x$ we may suppose that $\Theta=(1,0, \ldots, 0)$ so that

$$
\mathcal{L}(x)=x_{1} I+\sum_{j=2}^{n} F^{j} x_{j}=x_{1} I+L\left(x^{\prime}\right)
$$

where $F^{j} \in M^{s}(m, \mathbf{R}), x^{\prime}=\left(x_{2}, \ldots, x_{n}\right)$ and $\left\{F^{2}, \ldots, F^{n}, I\right\}$ are linearly independent.

Theorem 2.1 Assume that every characteristic of $\mathcal{L}(x)$ of order less than $m$ is non degenerate. Suppose that $\operatorname{det} S_{\mathcal{L}}(x)$ satisfies (1.2). Then for every hyperbolic perturbation $\mathcal{P}(x)=\mathcal{L}(x)+R(x)$ of $\mathcal{L}(x)$ we can find real analytic $A(x), B(x)$ defined near the origin with $A(0) B(0)=I$ so that

$$
A(x) \mathcal{P}(x) B(x)
$$

becomes symmetric.

Proof. By a preparation theorem for systems proved in [3, Theorem 4.3], generalizing the Weierstrass preparation theorem, one can write

$$
\mathcal{P}(x+\lambda \Theta)=C(x, \lambda)(\lambda I+\mathcal{Q}(x))
$$

where $C(x, \lambda)$ is real analytic near $(0,0), \operatorname{det} C(0,0) \neq 0$ and $\mathcal{Q}(x)$ is real analytic with values in $M(m, \mathbf{R}), \mathcal{Q}(0)=O$. Comparing the first order term in the Taylor expansion at $(0,0)$ of both sides we see that $C(0,0)=I$ and $\mathcal{Q}(x)=\mathcal{L}(x)+\tilde{R}(x)$ where $\tilde{R}(x)=O\left(|x|^{2}\right)$. Taking $x^{\prime}=0, \lambda=-x_{1}$ we get that $O=C\left(x_{1}, 0,-x_{1}\right) \tilde{R}\left(x_{1}, 0\right)$ and hence $\tilde{R}\left(x_{1}, 0, \ldots, 0\right)=O$. Since

$$
C(x, 0)^{-1} \mathcal{P}(x)=\mathcal{L}(x)+\tilde{R}(x)
$$

it is enough to study a perturbation term $R(x)$ which verifies $R\left(x_{1}, 0, \ldots\right.$, $0)=O$. We also note that $C(\epsilon \Theta, 0)^{-1} \mathcal{P}(\epsilon \Theta)=\epsilon I$ for small $\epsilon$. We set

$$
P\left(x^{\prime}, x_{1}\right)=L\left(x^{\prime}\right)+R\left(x_{1}, x^{\prime}\right), \quad L\left(x^{\prime}\right)=\sum_{j=2}^{n} F^{j} x_{j}
$$

where $S_{L}\left(x^{\prime}\right)$ verifies the assumption (1.2) because $\mathcal{L}(x)-L\left(x^{\prime}\right)=x_{1} I$. Introducing the polar coordinates $x^{\prime}=r \omega$, we blow up $P\left(x^{\prime}, x_{1}\right)$ at $x^{\prime}=0$ so that $r^{-1} P\left(r \omega, x_{1}\right)$ will be studied. We first show that, for every fixed 
$\omega \neq 0$, there is a real analytic positive definite $H_{\omega}\left(r, \theta, x_{1}\right)$ with diagonal elements 1 defined near $(0, \omega, 0)$ such that

$$
P\left(r \theta, x_{1}\right) H_{\omega}\left(r, \theta, x_{1}\right)=H_{\omega}\left(r, \theta, x_{1}\right)^{t} P\left(r \theta, x_{1}\right) .
$$

To prove the above assertion we can follow the same proof of Proposition 4.3 in [9] except for that of Lemma 4.7 in [9] which was proved assuming that $x=0$ is non-degenerate. We examine that the assertion of Lemma 4.7 holds under the assumptions of Theorem 2.1. We fix $\omega \neq 0$ and take an orthogonal $T_{0}$ so that $T_{0}^{-1} L(\omega) T_{0}=\bigoplus_{i=1}^{p} \lambda_{i} I_{s_{i}}$ just as in the proof of Proposition 4.3. Set $\tilde{L}(\theta)=T_{0}^{-1} L(\theta) T_{0}=\left(\tilde{L}_{i j}(\theta)\right)_{1 \leq i, j \leq p}$ and

$$
\tilde{F}^{j}=T_{0}^{-1} F^{j} T_{0}=\left(\tilde{F}_{k l}^{j}\right)_{1 \leq k, l \leq p}, \quad \tilde{L}_{i i}(\theta)=\sum_{j=2}^{n} \tilde{F}_{i i}^{j} \theta_{j}
$$

where the block decomposition corresponds to that of $\oplus \lambda_{i} I_{s_{i}}$. Then it is easy to see that to prove the assertion of Lemma 4.7 it is enough to show the following.

Lemma $2.2\left\{I_{s_{i}}, \tilde{F}_{i i}^{j}\right\}$ span $M^{s}\left(s_{i}, \mathbf{R}\right)$.

Proof. Let $\tilde{\mathcal{L}}(x)=T_{0}^{-1} \mathcal{L}(x) T_{0}$. Since $\left(x_{1}, x^{\prime}\right)=\left(-\lambda_{i}, \omega\right)$ is a characteristic of $\tilde{\mathcal{L}}(x)$ of order less than $m$ it is non-degenerate by assumption. It is clear that the localization of $\tilde{\mathcal{L}}(x)$ at $\left(-\lambda_{i}, \omega\right)$ is

$$
\tilde{\mathcal{L}}_{\left(-\lambda_{i}, \omega\right)}(x)=x_{1} I_{s_{i}}+\sum_{j=2}^{n} \tilde{F}_{i i}^{j} x_{j}
$$

because $\tilde{\mathcal{L}}\left(-\lambda_{i}, \omega\right)$ is diagonal. Noting that the non-degeneracy of characteristics is invariant under changes of basis for $\mathbf{C}^{m}$ we conclude that the matrices $\left\{I_{s_{i}}, \tilde{F}_{i i}^{j}\right\}$ span $M^{s}\left(s_{i}, \mathbf{R}\right)$ since the image $\tilde{\mathcal{L}}_{\left(-\lambda_{i}, \omega\right)}$ is $s_{i}$-dimensional. This proves the assertion.

Thus we get $H_{\omega}\left(r, \theta, x_{1}\right)$ near every $\omega \neq 0$ verifying $[2.3)$ with diagonal elements 1 . Since $\operatorname{det} S_{L}(\theta) \neq 0$ on a dense subset then $H_{\omega}$ can be continued analytically to a neighborhood of $\{0\} \times S^{n-2} \times\{0\}$ yielding $H\left(r, \theta, x_{1}\right)$ which verifies (2.3) there (see Lemma 4.8 in [9]). We then show that there is a real analytic $G\left(x^{\prime}, x_{1}\right)$ defined near the origin such that

$$
H\left(r, \theta, x_{1}\right)=G\left(r \theta, x_{1}\right), \quad G(0)=I
$$

which proves that $T(x)^{-1} P(x) T(x)$ becomes symmetric with $T(x)=G(x)^{1 / 2}$. 
Taking $A(x)=T(x)^{-1} C(x, 0)^{-1}, B(x)=T(x)$ we obtain Theorem 2.1. Here we note that $A(\epsilon \Theta) \mathcal{P}(\epsilon \Theta) B(\epsilon \Theta)=\epsilon I$ for small $\epsilon$. To see $(2.4)$ we make the following observation. Let $f(\theta), g(\theta)$ be homogeneous polynomials in $\theta$ of degree $p, q$ respectively where $p \geq q$. Let

$$
g(\theta)=\prod_{j=1}^{s} g_{j}(\theta)^{r_{j}}
$$

be the irreducible factorization of $g(\theta)$ in $\mathbf{R}[\theta]$. We assume that $f(\theta) / g(\theta)$ is $C^{\infty}$ apart from the origin and that $V_{j}=\left\{\theta \mid g_{j}(\theta)=0\right\}, 1 \leq j \leq s$ contains a regular point. Then applying Lemma 2.5 in [6] repeatedly, we conclude that $f(\theta) / g(\theta)$ is a homogeneous polynomial in $\theta$ of degree $p-q$.

Then, in the proof of Proposition 4.5 in [9], replacing Lemma 4.9 by the assumption (1.2) and the argument applying Lemma 2.5 in [6] by the above observation, we conclude (2.4) easily.

Since the non-degeneracy of characteristics is invariant under orthogonal changes of basis for $\mathbf{C}^{m}$ we have

Corollary 2.3 Assume that every characteristic of $\mathcal{L}(x)$ of order less than $m$ is non-degenerate and there is an orthogonal $T \in O(m)$ such that $\operatorname{det} S_{T^{-1} \mathcal{L} T}(x)$ verifies (1.2). Then the same conclusion as in Theorem 2.1 holds.

Remark. The condition (1.2) is not invariant under orthogonal changes of basis for $\mathbf{C}^{m}$. Let

$$
\mathcal{L}(x)=x_{1} I_{2}+\left(\begin{array}{cc}
0 & x_{2} \\
x_{2} & 0
\end{array}\right) .
$$

Then it is obvious that $\operatorname{det} S_{\mathcal{L}}(x)=0$. But it is easy to see that there is an orthogonal $T \in O(2)$ so that $\operatorname{det} S_{T^{-1} \mathcal{L} T}(x)$ verifies (1.2).

We remark here that the definition of non-degenerate characteristics given here is equivalent to that used in the previous papers [4], [2] for double characteristics. Let

$$
\mathcal{L}(x)=x_{1} I+L\left(x^{\prime}\right), \quad x^{\prime}=\left(x_{2}, \ldots, x_{n}\right),
$$

where $L\left(x^{\prime}\right)$ is real analytic with values in $M(m, \mathbf{R})$ defined near $x^{\prime}=\bar{x}^{\prime}$ which is not necessarily linear in $x^{\prime}$.

Lemma 2.4 Assume that all eigenvalues of $L\left(x^{\prime}\right)$ are real near $x^{\prime}=\bar{x}^{\prime}$. 
Let $\bar{x}=\left(\bar{x}_{1}, \bar{x}^{\prime}\right)$ be a double characteristic of $\mathcal{L}(x)$. Then $\bar{x}$ is non degenerate if and only if

$\operatorname{dimKer} \mathcal{L}(\bar{x})=2$ and $\operatorname{rankHess} h(\bar{x})=3$

where $h(x)=\operatorname{det} \mathcal{L}(x)$.

Proof. Take a constant matrix $T$ so that

$$
T^{-1} \mathcal{L}(\bar{x}) T=\left(\begin{array}{cc}
A & O \\
O & G
\end{array}\right)
$$

where $G$ is a non singular matrix of order $m-2$ and the two eigenvalues of $A$ are zero. Assume that $\operatorname{dimKer} \mathcal{L}(\bar{x})=2$ and $\operatorname{rankHess} h(\bar{x})=3$. Then it follows that $A=O$ and hence $\operatorname{Ker} \mathcal{L}(\bar{x}) \cap \operatorname{Im} \mathcal{L}(\bar{x})=\{0\}$. Let $\mathcal{L}_{\bar{x}}(x)$ be the localization of $\mathcal{L}(x)$ at $\bar{x}$. Denoting $T^{-1} \mathcal{L}(x) T=\left(L_{i j}(x)\right)_{1 \leq i, j \leq 2}$ we get $L_{11}(\bar{x}+\mu x)=\mu\left(\mathcal{L}_{\bar{x}}(x)+O(\mu)\right)$ as $\mu \rightarrow 0$. Then it follows that

$$
h(\bar{x}+x)=\operatorname{det} \mathcal{L}(\bar{x}+x)=(\operatorname{det} G) \operatorname{det} \mathcal{L}_{\bar{x}}(x)+O\left(|x|^{3}\right)
$$

as $x \rightarrow 0$. Since $\mathcal{L}_{\bar{x}}(x)$ is a $2 \times 2$ hyperbolic system and $\operatorname{rankHess} \operatorname{det} \mathcal{L}_{\bar{x}}(0)=$ 3 by (2.5) then it can be symmetrized by a constant matrix by Lemma 4.1 in [7]. In particular $\mathcal{L}_{\bar{x}}(x)$ is diagonalizable for every $x$ and $\operatorname{dim}\left\{\mathcal{L}_{\bar{x}}(x) \mid x \in\right.$ $\left.\mathbf{R}^{n}\right\}=3$. Conversely we assume that $\bar{x}$ is non degenerate in the sense of Definition 2.1. From $\operatorname{Ker} \mathcal{L}(\bar{x}) \cap \operatorname{Im} \mathcal{L}(\bar{x})=\{0\}$ it follows that $A=O$ and hence $\operatorname{dimKer} \mathcal{L}(\bar{x})=2$. Since $\mathcal{L}_{\bar{x}}(x)$ is diagonalizable and $\operatorname{dim} \mathcal{L}_{\bar{x}}=3$ then $\mathcal{L}_{\bar{x}}(x)$ is symmetrizable (see [2]). Thus rankHess $\operatorname{det} \mathcal{L}_{\bar{x}}(0)=3$ and hence $\operatorname{rankHess} h(\bar{x})=3$ by $(2.5)$.

\section{Non-degenerate characteristics for symmetric systems}

For symmetric systems with constant coefficients the description of non degeneracy of characteristics becomes simple. Let $\mathcal{L}(x)$ be

$$
\mathcal{L}(x)=\sum_{j=1}^{n} A_{j} x_{j}
$$

where $A_{j} \in M^{s}(m, \mathbf{R})$. We denote by $M_{k}^{s}(m, \mathbf{R})$ the set of all $A \in M^{s}(m, \mathbf{R})$ with rank $m-k$. Then we have

Lemma 3.1 Let $\bar{x}$ be a characteristic of $\mathcal{L}(x)$ of order $k$. Then $\bar{x}$ is nondegenerate if and only if the range $\mathcal{L}$ intersects $M_{k}^{s}(m, \mathbf{R})$ at $\mathcal{L}(\bar{x})$ transversally. 
Proof. Since $\mathcal{L}(\bar{x})$ and $\mathcal{L}_{\bar{x}}(x)$ are symmetric, the conditions (1) and (3) in Definition 2.1 are automatically satisfied. Without restrictions we may assume that $\bar{x}=(0, \ldots, 0,1)$. Then $A_{n}$ is of rank $m-k$. We can make an orthogonal transformation of the matrices to attain that with a block matrix notation

$$
A_{n}=\left(\begin{array}{ll}
O & O \\
O & G
\end{array}\right)
$$

where $G$ is a $(m-k) \times(m-k)$ non-singular matrix. The tangent space of $M_{k}^{s}(m, \mathbf{R})$ at $A_{n}$ consists of matrices of the form

$$
\left(\begin{array}{ll}
O & * \\
* & *
\end{array}\right)
$$

with the corresponding block decomposition. On the other hand, with the same block decomposition of $\mathcal{L}(x)$

$$
\mathcal{L}(x)=\left(\begin{array}{ll}
L_{11}(x) & L_{12}(x) \\
L_{21}(x) & L_{22}(x)
\end{array}\right)
$$

it is clear that $\mathcal{L}_{\bar{x}}(x)=L_{11}(x)$. Thus the transversality of intersection means that $\operatorname{dim} L_{11}=d(k)$ that is, $\operatorname{dim} \mathcal{L}_{\bar{x}}=d(k)$ and hence $\bar{x}$ is non-degenerate. The converse follows in the same way.

Taking Lemma 2.4 into account one sees that Lemma 3.1 generalizes Lemma 3.2 in [4].

We continue to study non-degenerate characteristics for $\mathcal{L}(x)$ in (2.2). We start with the special case that $\operatorname{dim} \mathcal{L}=d(m)-1$. Since $\mathcal{L}$ has codimension one in $M^{s}(m, \mathbf{R})$ then $\mathcal{L}$ is defined by

$$
\mathcal{L}: \operatorname{tr}(A X)=0, \quad X=\left(x_{i j}\right), \quad x_{i j}=x_{j i}
$$

with some $A \in M^{s}(m, \mathbf{R})$. Note that $\operatorname{tr} A=0$ because $\mathcal{L}$ contains the identity. Now we have

Proposition 3.2 Assume that $\mathcal{L}$ is given by $(3.2)$ with $A \in M^{s}(m, \mathbf{R})$ and that the rank of $A$ is greater than $k$. Then every characteristic of order $k$ of $\mathcal{L}(x)$ is non-degenerate.

Proof. Let $\bar{x}$ be a characteristic of order $k$ of $\mathcal{L}(x)$ and hence $H=\mathcal{L}(\bar{x}) \in$ $\mathcal{L} \cap M_{k}^{s}(m, \mathbf{R})$. Here we note that $\operatorname{dim} T_{H} M_{k}^{s}(m, \mathbf{R})=d(m)-d(k)$ which is seen by the proof of Lemma 3.1. To show $\bar{x}$ is non-degenerate it suffices 
to prove that

$$
\operatorname{dim}\left(\mathcal{L} \cap T_{H} M_{k}^{s}(m, \mathbf{R})\right)=d(m)-d(k)-1
$$

by Lemma 3.1. As in the proof of Lemma 3.1, considering $T^{-1} \mathcal{L} T$ with a suitable $T \in O(m)$ we may assume that

$$
H=\left(\begin{array}{ll}
O & O \\
O & G
\end{array}\right)
$$

where $G$ is a $(m-k) \times(m-k)$ non-singular matrix. Recalling that the tangent space $T_{H} M_{k}^{s}(m, \mathbf{R})$ is spanned by matrices of the form (3.1) we see that $\mathcal{L} \cap T_{H} M_{k}^{s}(m, \mathbf{R})$ consists of matrices of the form

$$
X=\left(\begin{array}{cc}
O & x_{i j} \\
x_{i j} & x_{i j}
\end{array}\right), \quad \operatorname{tr}(A X)=\sum_{k+1 \leq j, i \leq j}\left(2-\delta_{i j}\right) a_{i j} x_{i j}=0
$$

where $A=\left(a_{i j}\right)$ and $\delta_{i j}$ is the Kronecker's delta. Since $A$ is symmetric and the rank of $A$ is greater than $k$ by assumption then it follows that $\left(a_{i j}\right)_{k+1 \leq j, i \leq j} \neq O$. This proves $(3.3)$ and hence the assertion.

We turn to the general case that $1 \leq \operatorname{dim} \mathcal{L} \leq d(m)-1$.

Proposition 3.3 In the Grassmannian $G_{d(m), I}^{n}$ of $n$ dimensional subspaces of $M^{s}(m, \mathbf{R})$ containing the identity $I$, the subset for which every characteristic of order less than $m$ is non-degenerate is an open and dense subset.

Let $\mathbf{P}^{N}(\mathbf{R})$ be the $N$ dimensional real projective space and let $X \subset$ $\mathbf{P}^{N}(\mathbf{R})$ be a non-singular algebraic manifold of dimension $r$ and assume that $x_{0} \notin T_{x} X$ for all $x \in X$. Let us denote

$$
\tilde{G}_{N, x_{0}}^{s}=\left\{W \subset \mathbf{P}^{N}(\mathbf{R}) \mid W ; \text { linear space, } \operatorname{dim} W=s, x_{0} \in W\right\}
$$

and set $s^{\prime}=N-s$. Then we have

Lemma 3.4 A generic $W \in \tilde{G}_{N, x_{0}}^{s}$ intersects $X$ transversally.

Proof. ${ }^{1}$ Let $Y=\left\{(x, W) \in X \times \tilde{G}_{N, x_{0}}^{s} \mid x \in W\right\}$ and denote by $p_{1}, p_{2}$ the projections onto $X$ and $\tilde{G}_{N, x_{0}}^{s}$ respectively. Note that $\operatorname{dim} Y=s^{\prime} s-s^{\prime}+r$ and $\operatorname{dim} \tilde{G}_{N, x_{0}}^{s}=s^{\prime} s$. Then if $r<s^{\prime}$ a generic $W \in \tilde{G}_{N, x_{0}}^{s}$ does not intersect

\footnotetext{
${ }^{1}$ The author owes this simple proof to A.Gyoja
} 
$X$ and hence the result. Thus it is enough to study the case $r \geq s^{\prime}$. Let us set

$$
Z=\left\{(x, W) \in Y \mid \operatorname{dim}\left(T_{x} X+W\right) \leq N-1\right\}
$$

It is not difficult to see that

$$
\operatorname{dim}\left(p_{1} \mid Z\right)^{-1}(x)=s s^{\prime}-r-1, \quad x \in X
$$

so that $\operatorname{dim} Z=s s^{\prime}-1=\operatorname{dim} \tilde{G}_{N, x_{0}}^{s}-1$. Thus for every $W$ belonging to the open dense subset $\tilde{G}_{N, x_{0}}^{s} \backslash \overline{p_{2}(Z)}, W$ intersects $X$ transversally. This proves the assertion.

Proof of Proposition 3.3 Take $X$ and $\tilde{G}_{N, x_{0}}^{s}$ as the projective spaces $M_{k}^{s}(m, \mathbf{R})^{p r}$ and $\left(G_{d(m), I}^{s+1}\right)^{p r}$ based on $M_{k}^{s}(m, \mathbf{R})$ and $G_{d(m), I}^{s+1}$ respectively. Applying Lemma 3.4 with $N=d(m)-1, r=N-d(k), x_{0}=I$ we get the desired result.

\section{Condition (1.2)}

As mentioned in Introduction we study $S_{\mathcal{L}}(x)$ for symmetric $\mathcal{L}(x)$ when $\operatorname{dim} \mathcal{L}=d(m)-\nu$ where $1 \leq \nu \leq m-3$. We first examine a matrix representation of $S_{\mathcal{L}}(x)$. Let

$$
F_{m}=\left\{H=\left(h_{i j}\right) \in M^{s}(m, \mathbf{R}) \mid h_{i i}=0\right\}
$$

then $S_{\mathcal{L}}(x)$ is defined as the linear map between two $d(m-1)$-dimensional linear subspaces $F_{m}$ and $M^{a s}(m, \mathbf{R})$

$$
F_{m} \ni H \mapsto[\mathcal{L}(x), H]=K \in M^{a s}(m, \mathbf{R})
$$

where $M^{a s}(m, \mathbf{R})$ denotes the set of all real anti-symmetric matrices of order $m$. Let us write

$$
\mathcal{L}(x)=\left(\phi_{j}^{i}(x)\right)_{1 \leq i, j \leq m}, \quad \phi_{j}^{i}(x)=\phi_{i}^{j}(x) .
$$

For $H \in F_{m}$ we write $\check{H}={ }^{t}\left(h_{12}, h_{13}, h_{23}, h_{14}, h_{24}, h_{34}, \ldots, h_{m-1 m}\right) \in$ $\mathbf{R}^{d(m-1)}$. Then the equation $[\mathcal{L}(x), H]=K$ can be written as

$$
S_{\mathcal{L}}(x) \check{H}=\check{K}
$$


where $S_{\mathcal{L}}(x)$ is a $d(m-1) \times d(m-1)$ matrix. For instance when $m=3$ we have

$$
S_{\mathcal{L}}(x)=\left(\begin{array}{ccc}
\phi_{1}^{1}(x)-\phi_{2}^{2}(x) & -\phi_{3}^{2}\left(x^{\prime}\right) & \phi_{3}^{1}\left(x^{\prime}\right) \\
-\phi_{3}^{2}\left(x^{\prime}\right) & \phi_{1}^{1}(x)-\phi_{3}^{3}(x) & \phi_{2}^{1}\left(x^{\prime}\right) \\
-\phi_{3}^{1}\left(x^{\prime}\right) & \phi_{2}^{1}\left(x^{\prime}\right) & \phi_{2}^{2}(x)-\phi_{3}^{3}(x)
\end{array}\right)
$$

We turn to the case $\mathcal{L}(x)$ is a $m \times m$ matrix. Let

$$
\mathcal{L}(x)=\left(\begin{array}{cc}
L(x) & l\left(x^{\prime}\right) \\
{ }^{t} l\left(x^{\prime}\right) & \phi_{m}^{m}(x)
\end{array}\right)
$$

where $l\left(x^{\prime}\right)={ }^{t}\left(\phi_{m}^{1}\left(x^{\prime}\right), \ldots, \phi_{m}^{m-1}\left(x^{\prime}\right)\right)$ and $L(x)$ stands for $\mathcal{L}(x)$ in (4.1) with $m-1$. For $H \in F_{m}$ and $K \in M^{a s}(m, \mathbf{R})$ we write

$$
H=\left(\begin{array}{cc}
H_{1} & h \\
{ }^{t} h & 0
\end{array}\right), \quad K=\left(\begin{array}{cc}
K_{1} & k \\
{ }^{t} k & 0
\end{array}\right)
$$

with $H_{1} \in F_{m-1}, K_{1} \in M^{a s}(m-1, \mathbf{R})$ and $h={ }^{t}\left(h_{1 m}, \ldots, h_{m-1 m}\right)$. Then it is easy to see that the equation $[\mathcal{L}(x), H]=K$ is written as

$$
\left(\begin{array}{cc}
S_{L}(x) & c(l) \\
c^{\prime}(l) & L(x)-\phi_{m}^{m} I
\end{array}\right)\left(\begin{array}{c}
\check{H}_{1} \\
h
\end{array}\right)=\left(\begin{array}{c}
\check{K}_{1} \\
k
\end{array}\right)=\check{K}
$$

and hence we get

$$
S_{\mathcal{L}}(x)=\left(\begin{array}{cc}
S_{L}(x) & c(l) \\
c^{\prime}(l) & L(x)-\phi_{m}^{m} I
\end{array}\right) .
$$

Our aim in this section is to prove

Proposition 4.1 Assume that $1 \leq \nu \leq m-3$. Then in the Grassmannian $G_{d(m), I}^{d(m)-\nu}$, the subset of $\mathcal{L}$ for which the condition $(1.2)$ is fulfilled for $T^{-1} \mathcal{L} T$ with some $T \in O(m)$ is an open and dense subset.

We first give a parametrization of the Grassmannian $G_{d(m), I}^{n}$ of $n$ dimensional subspaces of $M^{s}(m, \mathbf{R})$ containing the identity. Take a map

$$
\sigma:\{1, \ldots, \nu\} \rightarrow\{(i, j) \mid 1 \leq i \leq j \leq m,(i, j) \neq(m, m)\}
$$

which is injective. Denote by $U_{\sigma}$ the set of all $\nu$-tuples of $m \times m$ symmetric matrices $A=\left(A_{1}, \ldots, A_{\nu}\right)$ such that $\operatorname{tr} A_{j}=0$ and the element $\sigma(k)$ of $A_{j}$ 
is zero unless $k=j$ and the element $\sigma(j)$ of $A_{j}$ is 1 . Let

$$
\begin{aligned}
& \phi_{\sigma}: U_{\sigma} \ni A \mapsto \mathcal{L}, \\
& \mathcal{L}=\left\{X \in M^{s}(m, \mathbf{R}) \mid \operatorname{tr}\left(A_{j} X\right)=0,1 \leq j \leq \nu\right\}
\end{aligned}
$$

and set $\Omega_{\sigma}=\phi_{\sigma}\left(U_{\sigma}\right)$ then with all such injective $\sigma,\left(\phi_{\sigma}^{-1}, \Omega_{\sigma}\right)$ give charts of the Grassmannian $G_{d(m), I^{\prime}}^{n}$. We set $\triangle=\{(i, i) \mid 1 \leq i \leq m\}$ and let $1 \leq k \leq m-1$. We first remark that

Lemma 4.2 Assume that $1 \leq k \leq m-1$. Then there are finitely many $S_{1}, \ldots, S_{N} \in O(m)$ such that for any $\mathcal{L} \in G_{d(m), I}^{d(m)-k}$ one can find $S_{i}$ so that $S_{i}^{-1} \mathcal{L} S_{i} \in \Omega_{\sigma}$ with some $\sigma$ verifying $\sigma(\{1, \ldots, k\}) \cap \triangle=\emptyset$.

Proof. In this proof we denote $|C|=\max _{i, j}\left|c_{i j}\right|$ for a matrix $C=\left(c_{i j}\right)$. Let $T_{p q}(\epsilon)$ be the orthogonal matrix obtained replacing $p$-th and $q$-th, $p<q$, rows of the identity matrix by

$$
\begin{aligned}
& (0, \ldots, 0, f(\epsilon), 0, \ldots, 0, \epsilon, 0, \ldots, 0) \\
& (0, \ldots, 0,-\epsilon, 0, \ldots, 0, f(\epsilon), 0, \ldots, 0)
\end{aligned}
$$

where $\epsilon^{2}+f(\epsilon)^{2}=1$. We show that it is enough to take $\left\{S_{i}\right\}$ as the set of all $m$ times compositions of $I$ and $T_{p q}\left(\epsilon_{i}\right), \epsilon_{i}=\left(C_{i} m^{2^{i-1}}\right)^{-1}, i=1, \ldots, m$, where $C_{1}<C_{2}<\cdots<C_{m}$ will be chosen suitably. Let $\mathcal{L} \in G_{d(m), I}^{d(m)-k}$ and let $A_{1}, \ldots, A_{k}$ define $\mathcal{L}$ so that $\mathcal{L}$ consists of all $X \in M^{s}(m, \mathbf{R})$ such that $\operatorname{tr}\left(A_{j} X\right)=0,1 \leq j \leq k$ where $A_{j}$ are linearly independent and $\operatorname{tr} A_{j}=0$. We first note that we may assume $(H)_{\mu}$ : there is an injective $\tau:\{1, \ldots, \mu\} \rightarrow\{(i, j) \mid 1 \leq i<j \leq m\}$ such that the element $\tau(i)$ of $A_{j}$ is zero unless $i=j$, the element $\tau(j)$ of $A_{j}$ is $1,\left|A_{j}\right| \leq a_{\mu} m^{2^{\mu-1}}$ for $1 \leq j \leq \mu$ and $A_{\mu+1}, \ldots, A_{k}$ are diagonal where $a_{1}=1, a_{\mu+1}=B a_{\mu} C_{\mu}$ with a fixed large $B$. In fact if some $A_{j}$ has a non-zero off diagonal element we may assume that the off diagonal element $\tau(1)$ of $A_{1}$ is 1 and $\left|A_{1}\right| \leq 1$. Replacing $A_{j}$ by $A_{j}-\alpha_{j} A_{1}, j \neq 1$, with suitable $\alpha_{j}$ one can assume that the element $\tau(1)$ of $A_{j}$ is zero if $j \neq 1$. A repetition of this argument gives the assertion. If $\mu=k$ then $\tau(\{1, \ldots, k\}) \cap \triangle=\emptyset$ and there is nothing to prove. Then we may assume that $\mu \leq k-1$. Let $A_{\mu+1}=\operatorname{diag}\left(\lambda_{1}, \ldots, \lambda_{m}\right)$. Since $\operatorname{tr} A_{\mu+1}=0$ it is easy to see that there are at least $m-1$ pairs $(i, j)$, $i<j$ such that

$$
3\left|\lambda_{i}-\lambda_{j}\right| \geq\left|\lambda_{r}\right|, \quad r=1, \ldots, m .
$$


Since $\mu \leq m-2$ there exists such a $(p, q)$ with $(p, q) \notin \tau(\{1, \ldots, \mu\})$. Let us set

$$
A_{j}\left(\epsilon_{\mu}\right)=T_{p q}\left(\epsilon_{\mu}\right)^{-1} A_{j} T_{p q}\left(\epsilon_{\mu}\right), \quad 1 \leq j \leq k
$$

and note that $\left|A_{j}\left(\epsilon_{\mu}\right)-A_{j}\right| \leq B_{1} a_{\mu} C_{\mu}^{-1}, 1 \leq j \leq \mu$. Choose $C_{\mu}$ so that $a_{\mu} C_{\mu}^{-1}$ is small enough then taking $\tilde{A}_{j}\left(\epsilon_{\mu}\right)=\sum_{i=1}^{\mu} c_{j i} A_{i}\left(\epsilon_{\mu}\right), 1 \leq j \leq \mu$, with a non-singular $C=\left(c_{j i}\right)$ we may suppose that the element $\tau(i)$ of $\tilde{A}_{j}\left(\epsilon_{\mu}\right)$ is zero unless $i=j$ and the element $\tau(j)$ of $\tilde{A}_{j}\left(\epsilon_{\mu}\right)$ is 1 and $\left|\tilde{A}_{j}\left(\epsilon_{\mu}\right)\right| \leq 2\left|A_{j}\right|$. Note that the off diagonal elements of $A_{\mu+1}\left(\epsilon_{\mu}\right)$ are zero except for $(p, q)$, $(q, p)$ elements which are $\epsilon_{\mu} f\left(\epsilon_{\mu}\right)\left(\lambda_{q}-\lambda_{p}\right)$. Set

$$
\tilde{A}_{\mu+1}\left(\epsilon_{\mu}\right)=\left\{\epsilon_{\mu} f\left(\epsilon_{\mu}\right)\left(\lambda_{q}-\lambda_{p}\right)\right\}^{-1} A_{\mu+1}\left(\epsilon_{\mu}\right)
$$

and hence $\left|\tilde{A}_{\mu+1}\left(\epsilon_{\mu}\right)\right| \leq B_{2} C_{\mu} m^{2^{\mu-1}}$. Replacing $\tilde{A}_{j}\left(\epsilon_{\mu}\right)$ by $\tilde{A}_{j}\left(\epsilon_{\mu}\right)-$ $\alpha_{j} \tilde{A}_{\mu+1}\left(\epsilon_{\mu}\right)$ with suitable $\alpha_{j}$ we can attain that the element $\tau(\mu+1)=(p, q)$ of $\tilde{A}_{j}\left(\epsilon_{\mu}\right)$ is zero for $1 \leq j \leq \mu$ and $\left|\tilde{A}_{j}\left(\epsilon_{\mu}\right)\right| \leq a_{\mu+1} m^{2^{\mu}}, 1 \leq j \leq \mu+1$. By subtraction again we may suppose that $A_{j}\left(\epsilon_{\mu}\right), j \geq \mu+2$ are diagonal and then we get to $(H)_{\mu+1}$. The rest of the proof is clear.

Proof of Proposition 4.1 We first assume that $\mathcal{L} \in \Omega_{\tau}$ with $\tau(\{1, \ldots, \nu\}) \cap$ $\triangle=\emptyset$ and let $A=\left(A_{1}, \ldots, A_{\nu}\right) \in U_{\tau}$ be the coordinate of $\mathcal{L}$. Let us denote

$$
\mathcal{L}(x)=\sum_{j=1}^{n} K_{j} x_{j}=\left(\phi_{j}^{i}(x)\right)
$$

where $K_{j}, 1 \leq j \leq n=d(m)-\nu$, is a basis for $\mathcal{L}$ and set $g(x)=\operatorname{det} S_{\mathcal{L}}(x)$. Let $J_{\tau}=\{(i, j) \mid 1 \leq i \leq j \leq m\} \backslash \tau(\{1, \ldots, \nu\})$ and note that $\phi_{j}^{i}(x)$, $(i, j) \in J_{\tau}$ are linearly independent and $\triangle \subset J_{\tau}$. With $A_{k}=\left(a_{i j}^{(k)}\right)$ it is clear that the equations $\phi_{j}^{i}(x)=0,(i, j) \in J_{\tau} \backslash \triangle$ and $\operatorname{tr}\left(A_{k} \mathcal{L}(x)\right)=0$ define a plane

$$
\sum_{j=1}^{m} a_{j j}^{(k)} \phi_{j}^{j}(x)=\sum_{j=1}^{m-1} a_{j j}^{(k)}\left(\phi_{j}^{j}(x)-\phi_{m}^{m}(x)\right)=0, \quad 1 \leq k \leq \nu
$$

and $S_{\mathcal{L}}(x)$ is diagonal on the plane with the determinant

$$
g(x)=\prod_{1 \leq i<j \leq m}\left(\phi_{i}^{i}(x)-\phi_{j}^{j}(x)\right) .
$$

We show that there is a polynomial $\pi(A)$ in $a_{j j}^{(k)}, 1 \leq k \leq \nu, 1 \leq j \leq m-1$ 
such that if $\pi(A) \neq 0$ then no two $\phi_{i}^{i}(x)-\phi_{j}^{j}(x), i<j$ are proportional on the plane (4.4). To simplify notation we write $y_{i}$ for $\phi_{i}^{i}(x)-\phi_{m}^{m}(x)$ so that

$$
g(y)=\prod_{1 \leq i<j \leq m-1}\left(y_{i}-y_{j}\right) y_{1} \cdots y_{m-1}
$$

provided that $y \tilde{A}=0$ where $y=\left(y_{1}, \ldots, y_{m-1}\right)$ and $\tilde{A}=\left(a_{j j}^{(k)}\right)$ which is a $(m-1) \times \nu$ matrix. Suppose that some two $y_{i}-y_{j}$ are proportional on the plane $y \tilde{A}=0$ and hence $(b, y)=0$ with some $b \in \mathbf{R}^{m-1}$ for every $y$ with $y \tilde{A}=0$. Then it is clear that $\operatorname{rank}(\tilde{A}, b)=\operatorname{rank} \tilde{A}$. Note that at most two components of $b$ are the constant of the proportionality $c$ and the other components are either 0 or 1 (at most two 1 appear). Take a $(\nu+1) \times(\nu+1)$ submatrix of $(\tilde{A}, b)$ and expand the determinant with respect to the last column. Equating the determinant to zero we get a linear relation of $\nu$-minors of $\tilde{A}$ with coefficients which are either 1 or the proportional constant $c$. Since $\nu+1 \leq m-2$ we have at least $m-1$ such linear relations. Elimination of $c$ gives a quadratic equation in $\nu$-minors of $\tilde{A}$. Denote this equation by $\pi(A)=0$. Then we conclude that the rank of the matrix $(\tilde{A}, b)$ is $\nu+1$ if $\pi(A) \neq 0$. This shows that no two $y_{i}-y_{j}$ are proportional if $\pi(A) \neq 0$.

Let $g(x)=\prod g_{j}(x)^{r_{j}}$ be the irreducible factorization in $\mathbf{R}[x]$. Without restrictions we may assume that the plane $y \tilde{A}=0$ is given by $y_{b}=f\left(y_{a}\right)$, after a linear change of coordinates $y$ if necessary, where $y=\left(y_{a}, y_{b}\right)$ is a partition of the coordinates $y$. Then we have

$$
\prod g_{j}\left(y_{a}, f\left(y_{a}\right)\right)^{r_{j}}=\prod p_{i}\left(y_{a}\right)
$$

where $p_{i}\left(y_{a}\right)$ are linear in $y_{a}$ and no two $p_{i}\left(y_{a}\right)$ are proportional if $\pi(A) \neq 0$. Then it follows that $r_{j}=1$ and $g_{j}\left(y_{a}, f\left(y_{a}\right)\right)$ is a product of some $p_{i}\left(y_{a}\right)$ 's:

$$
g_{j}\left(y_{a}, f\left(y_{a}\right)\right)=\prod_{i \in I_{j}} p_{i}\left(y_{a}\right) .
$$

From this it is obvious that $\left\{g_{j}\left(y_{a}, f\left(y_{a}\right)\right)=0\right\}$ contains a regular point. Then it follows that $\left\{g_{j}(x)=0\right\}$ contains a regular point. This shows that, in $U_{\tau}$, the set of $A$ such that $S_{\mathcal{L}}(x)$ does not verify (1.2) is contained in an algebraic set. We now study $\mathcal{L} \in \Omega_{\sigma}$ with $\sigma(\{1, \ldots, \nu\}) \cap \triangle \neq \emptyset$. By Lemma 4.2 there is $S_{i} \in O(m)$ such that $S_{i}^{-1} \mathcal{L} S_{i} \in \Omega_{\tau}$ with some $\tau$ verifying $\tau(\{1, \ldots, \nu\}) \cap \triangle=\emptyset$. Since $\left\{S_{i}\right\}$ is a finite set the proof is clear.

Proof of Theorem 1.1 Let $d(m)-m+3 \leq n \leq d(m)$. Then Theorem 1.1 
follows immediately from Propositions $3.3,4.1$ and Corollary 2.3 .

\section{A special case}

In this section we prove Theorem 1.2. Thus we assume $m=3$ throughout the section. Let $\mathcal{L} \in G_{6, I}^{n}$ for $n=4$ or 5 . With a basis $K_{j}$ for $\mathcal{L}, \mathcal{L}$ is the range of

$$
\mathcal{L}(x)=\sum_{j=1}^{n} K_{j} x_{j}
$$

We first study the case $n=5$.

Lemma 5.1 In the Grassmannian $G_{6, I}^{5}$, the subset of $\mathcal{L}$ for which the condition (1.2) is fulfilled for $T^{-1} \mathcal{L} T$ with some $T \in O(m)$ is an open and dense subset.

Proof. Let $A=A_{1} \in U_{\sigma}$ be the coordinate of $\mathcal{L}$ and assume that $\sigma(1) \cap$ $\triangle=\emptyset$ so that the diagonal elements of $\mathcal{L}(x)$ are linearly independent. Considering $T^{-1} \mathcal{L}(x) T$ with suitable permutation matrix $T$, if necessary, we may assume that $\sigma(1)=(1,2)$ so that with $\mathcal{L}(x)=\left(\phi_{j}^{i}(x)\right)$ we have from $\operatorname{tr}(A \mathcal{L}(x))=0$ that

$$
-2 \phi_{2}^{1}(x)=a_{11}\left(\phi_{1}^{1}-\phi_{3}^{3}\right)+a_{22}\left(\phi_{2}^{2}-\phi_{3}^{3}\right)+2 a_{13} \phi_{3}^{1}+2 a_{23} \phi_{3}^{2} .
$$

From (4.2), with simplified notations, it is enough to study

$$
S(x, y)=\left(\begin{array}{ccc}
x_{1}-x_{2} & -y_{1} & y_{2} \\
-y_{1} & x_{1} & \phi(x, y) \\
-y_{2} & \phi(x, y) & x_{2}
\end{array}\right)
$$

where $\phi(x, y)=a_{1} x_{1}+a_{2} x_{2}+b_{1} y_{1}+b_{2} y_{2}$. We show that if $a_{1}+a_{2} \neq 1$ and $4 a_{1} a_{2}-1 \neq 0$ then the condition (1.2) is fulfilled. We first assume that $x_{1} x_{2}-\phi(x, 0)^{2}$ is irreducible. Note that $g(x, y)=\operatorname{det} S(x)$ is then irreducible. Indeed if $g(x, y)$ were reducible so that $g(x, y)=h(x, y) k(x, y)$ then from $g(x, 0)=\left(x_{1}-x_{2}\right) \psi(x)$ with $\psi(x)=x_{1} x_{2}-\phi(x, 0)^{2}$ we may suppose that

$$
h(x, y)=\psi(x)+p(x, y), \quad k(x, y)=x_{1}-x_{2}+q(y)
$$

where $p(x, 0)=0, q(y)=\alpha y_{1}+\beta y_{2}$. Equating the coefficients of $y_{j}$ in both sides of $g(x, y)=h(x, y) k(x, y)$ we see that $\alpha \psi(x), \beta \psi(x)$ have a factor 
$x_{1}-x_{2}$ which implies that $q=0$. This gives $g(x, y)=h(x, y)\left(x_{1}-x_{2}\right)$ which is a contradiction. Thus $g$ is irreducible. It is clear that $\{g(x, 0)=0\}$ has a regular point and hence so does $\{g(x, y)=0\}$. This proves the assertion.

Assume now that $\psi(x)=x_{1} x_{2}-\phi(x, 0)^{2}$ is reducible. From the assumption $4 a_{1} a_{2}-1 \neq 0$ it follows that $\psi(x)$ has no multiple factor. Note that $a_{1}+a_{2} \neq \pm 1$ implies that $\psi(x)$ and $x_{1}-x_{2}$ are relatively prime. The rest of the proof is a repetition of the last part of the proof of Proposition 4.1 .

We turn to the case $n=4$. We show that

Lemma 5.2 Assume that $n=4$ and every double characteristic of $\mathcal{L}(x)$ is non degenerate. Then the condition $(1.2)$ is fulfilled for $T^{-1} \mathcal{L}(x) T$ with a suitable $T \in O(3)$.

Proof. Following the proof of Theorems 3.5 and 3.6 in [4] we choose a specific basis for $\tilde{\mathcal{L}}=T^{-1} \mathcal{L} T$ with suitably chosen $T \in O(3)$ and show that (1.2) is fulfilled for $\tilde{\mathcal{L}}$ using this basis. From the proof of Theorem 3.3 in [4], if every double characteristic of $\mathcal{L}$ is non-degenerate, then only two cases occur, that is $\mathcal{L}$ has either four non-degenerate double characteristics or two non-degenerate double characteristics.

We first treat the case that $\mathcal{L}$ has four non-degenerate characteristics. Choosing a suitable $T \in O(3)$ we see from [4] that $A^{ \pm}=\alpha_{ \pm} \otimes \alpha_{ \pm}$and $B^{ \pm}=$ $\beta_{ \pm} \otimes \beta_{ \pm}$is a basis for $\tilde{\mathcal{L}}=T^{-1} \mathcal{L} T$ where $\alpha_{ \pm}=(a, \pm a, 1), \beta_{ \pm}=(b, \pm b, 1)$ and $a \neq b, a b \neq 0$. Now we can write

$$
\tilde{\mathcal{L}}(x)=A^{+} x_{1}+A^{-} x_{2}+B^{+} x_{3}+B^{-} x_{4} .
$$

With $X=x_{1}+x_{2}, Y=x_{1}-x_{2}, Z=x_{3}+x_{4}, W=x_{3}-x_{4}$ we have

$$
\tilde{\mathcal{L}}=\left(\begin{array}{ccc}
a^{2} X+b^{2} Z & a^{2} Y+b^{2} W & a X+b Z \\
a^{2} Y+b^{2} W & a^{2} X+b^{2} Z & a Y+b W \\
a X+b Z & a Y+b W & X+Z
\end{array}\right) .
$$

Therefore it follows from (4.2) and (5.1) that

$$
S_{\tilde{\mathcal{L}}}=\left(\begin{array}{ccc}
0 & -a Y-b W & a X+b Z \\
-a Y-b W & c X+d Z & a^{2} Y+b^{2} W \\
-a X-b Z & a^{2} Y+b^{2} W & c X+d Z
\end{array}\right)
$$

where $c=a^{2}-1, d=b^{2}-1$. Let $\tilde{g}=\operatorname{det} S_{\tilde{\mathcal{L}}}$. On the plane $a^{2} Y+b^{2} W=0$, 
that is, if $W=-a^{2} Y / b^{2}=e Y$ we get

$$
\tilde{g}=(c X+d Z)(a X+b Z+(a+b e) Y)(a X+b Z-(a+b e) Y) .
$$

Note that $a+b e \neq 0$ because $a \neq b$ and no two factors in the right-hand side are proportional. Now, as the end of the proof of Proposition 4.2, it is easy to conclude that $\tilde{g}$ satisfies (1.2).

We next study the case $\mathcal{L}$ has two non-degenerate double characteristics. With a suitable $T \in O(3)$ we see that $\tilde{\mathcal{L}}=T^{-1} \mathcal{L} T$ contains $K^{ \pm}=\alpha_{ \pm} \otimes \alpha_{ \pm}$ with $\alpha_{ \pm}=(a, \pm a, 1), a \neq 0$, which are intersections with $M_{2}^{s}(3, \mathbf{R})$. Since $\tilde{\mathcal{L}}$ contains the identity, as the third basis element in $\tilde{\mathcal{L}}$, one can take $K_{3}$

$$
K_{3}=\left(\begin{array}{ccc}
0 & 0 & -2 a \\
0 & 0 & 0 \\
-2 a & 0 & 2\left(a^{2}-1\right)
\end{array}\right)
$$

because $K^{+}+K^{-}+K_{3}=2 a^{2} I$. The fourth basis element in $\tilde{\mathcal{L}}$ can then be chosen of the form

$$
K_{4}=\left(\begin{array}{ccc}
0 & 0 & 0 \\
0 & \lambda & \mu \\
0 & \mu & \nu
\end{array}\right)
$$

Thus with $X=x_{1}+x_{2}, Y=x_{1}-x_{2}, Z=x_{3}, W=x_{4}$ and $c=a^{2}-1$ the matrix $K^{+} x_{1}+K^{-} x_{2}+K_{3} x_{3}+K_{4} x_{4}$ can be written

$$
\tilde{\mathcal{L}}=\left(\begin{array}{ccc}
a^{2} X & a^{2} Y & a X-2 a Z \\
a^{2} Y & a^{2} X+\lambda W & a Y+\mu W \\
a X-2 a Z & a Y+\mu W & X+2 c Z+\nu W
\end{array}\right) .
$$

We examine if there are other double characteristics, that is, if $\tilde{\mathcal{L}}$ is of rank 1 for some $(X, Y, Z, W)$ with $Z^{2}+W^{2} \neq 0$. It is not difficult to see that six 2 -minors of (5.2) vanish for such $(X, Y, Z, W)$ if and only if the equation

$$
4 a^{2} Z^{2}+2\left(a^{2}+1\right) \lambda Z W+\left(\lambda \nu-\mu^{2}\right) W^{2}=0
$$

has a real solution $(Z, W) \neq(0,0)$. Thus in order that $\tilde{\mathcal{L}}$ has two nondegenerate double characteristics it is necessary and sufficient that

$$
4 a^{2} \lambda \nu>4 a^{2} \mu^{2}+\left(a^{2}+1\right)^{2} \lambda^{2} .
$$

In particular $\lambda$ and $\nu$ have the same signs. From (5.2) and (4.2) it follows 
that

$$
S_{\tilde{\mathcal{L}}}=\left(\begin{array}{ccc}
-\lambda W & -a Y-\mu W & a X-2 a Z \\
-a Y-\mu W & c X-2 c Z-\nu W & a^{2} Y \\
-a X+2 a Z & a^{2} Y & c X-2 c Z+(\lambda-\nu) W
\end{array}\right) .
$$

If $c \neq 0$ then we consider $\tilde{g}=\operatorname{det} S_{\tilde{\mathcal{L}}}$ on $W=0$ so that

$$
\tilde{g}=(c X-2 c Z)(a X-2 a Z+a Y)(a X-2 a Z-a Y) .
$$

The same argument as before proves that (1.2) is verified for $\tilde{g}$. If $c=0$ and hence $a^{2}=1$ then

$$
\begin{gathered}
\tilde{g}=W\left(-\nu(a X-2 a Z)^{2}+\lambda\left(\nu^{2}-\mu^{2}\right) \alpha^{-1} Y^{2}\right. \\
\left.\quad+(\lambda-\nu) \alpha\left(W-a \mu \alpha^{-1} Y\right)^{2}\right) \\
=W h(X, Y, Z, W)
\end{gathered}
$$

where $\alpha=\lambda \nu-\mu^{2}$. From (5.3) it follows that $\alpha>0$ and $\nu^{2}-\mu^{2}>0$ because $\nu^{2}+\lambda^{2} \geq \lambda \nu>\mu^{2}+\lambda^{2}$. Then the quadratic form $h$ is indefinite and hence $\{h=0\}$ contains a regular point. This proves the assertion.

Proof of Theorem 1.2 If $n=6$ then the assertion follows from Theorem 4.2 in [9]. If $n=5$, combining Proposition 3.3 and Lemma 5.1 we get the result by Corollary 2.3. Let $n=4$. Then by virtue of Proposition 3.3 and Lemma 5.2 one can apply Corollary 2.3 to get the assertion.

\section{References}

[1] Atiyah M.F., Bott R. and Gårding L., Lacunas for hyperbolic differential operators with constant coefficients, I. Acta Math. 124 (1970), 109-189.

[2] Bernardi E. and Nishitani T., Remarks on symmetrization of $2 \times 2$ systems and the characteristic manifolds. Osaka J. Math. 29 (1992), 129-134.

[3] Dencker N., Preparation theorems for matrix valued functions. Ann. Inst. Fourier 43 (1993), 865-892.

[4] Hörmander L., Hyperbolic systems with double characteristics. Comm. Pure Appl. Math.46 (1993), 261-301.

[5] John F., Algebraic conditions for hyperbolicity of systems of partial differential equations. Comm. Pure Appl. Math. 31 (1978), 787-793.

[6] Milnor J., Singular Points of Complex Hypersurfaces. Princeton University Press, Princeton, New Jersey, 1968.

[7] Nishitani T., On strong hyperbolicity of systems. in Res. Notes in Math. 158 Longman, London, 1987, pp. 102-114.

[8] Nishitani T., On localizations of a class of strongly hyperbolic systems. Osaka J. 
Math. 32 (1995), 41-69.

[9] Nishitani T., Symmetrization of hyperbolic systems with non-degenerate characteristics. J. Func. Anal. 132 (1995), 92-120.

[10] Vaillant J., Symétrisabilité des matrices localisées d'une matrice fortement hyperbolique. Ann. Scuo. Norm. Sup. Pisa 5 (1978), 405-427.

Department of Mathematics

Graduate School of Science

Osaka University

1-16 Machikaneyama

Toyonaka Osaka 560, Japan 